\title{
Regards croisés sur la cause première: Plotin, Porphyre, Victorinus, Saloustios, Proclus
}

\author{
Anca Vasiliu \\ CNRS, Centre «Léon Robin», Sorbonne Université
}

\section{$1 \quad$ Prémisses}

Théoriquement, remonter à la cause première est une démarche qui s'appuie nécessairement sur un présupposé: ce à partir de quoi on tente l'accès à la cause première doit en provenir pour pouvoir nous y conduire; doit, en outre, posséder la détermination de ce qu' il est en tant qu' effet de la cause première tout en révélant la raison en vue de laquelle il est l' effet de cette cause. Or la détermination substantielle, tout autant que la raison formelle et téléologique de ce qui provient, se projettent toutes deux sur la cause en la déterminant à leur tour: sur le plan causal d'une part, en affectant ce qui se trouve en condition de cause première, et sur le plan épistémique d'autre part, dès lors que seule la causalité conditionne dans ce cas la connaissance de ce qui accomplit ce rôle. En situation de primauté, la cause s' appuie donc sur l' effet autant que l'effet sur la cause, selon une perspective inversée et une symétrie inhabituelle pour une relation de type causal. Plus simplement dit: en considérant une cause comme première on rend la cause débitrice de son effet. Échappe-ton à ce présupposé en en stipulant un autre, à savoir que la cause première n' est cause que des choses premières, et que celles-ci n' obéissent pas nécessairement aux mêmes principes et raisons que toutes les autres, choses immanentes ou secondes?

L' affirmation que tout ce qui est provient d' une cause une et première - pro-

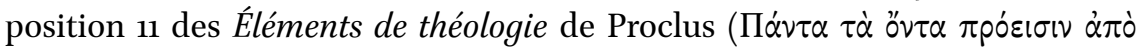

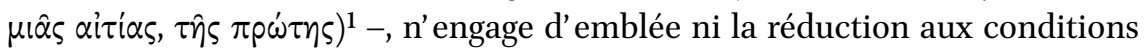
de possibilité pour la connaissance, ni la restriction du domaine d'action de la cause première à des étants premiers, voire à un seul étant qui se constituerait à son tour en cause seconde pour un autre étant ou genre d' étants de condition seconde. Il semble aller de soi, pour Proclus du moins, que c' est précisément

1 Proclus, The Elements of Theology, éd. E.R. Dodds.

(C) KONINKLIJKE BRILL NV, LEIDEN, THE NETHERLANDS, 2022 | DOI:10.1163/9789004501331_005 
la totalité qui est engagée par la cause première. Mais l' engagement de la totalité par une cause première peut s'entendre de deux manières: soit comme loi universelle (de quelque chose vient toute chose), soit comme expression d'une structure hiérarchique (la totalité n'étant pas homogène, les choses supérieures dépendent d'une cause première et sont à leur tour cause pour les choses inférieures - supérieur et inférieur n'indiquant pas une situation topique mais une différenciation ontologique eu égard à l' enchaînement d'une relation causale comprenant tout). À l'évidence, Proclus privilégie cette seconde option puisque les dix premières propositions fixent deux conditions complémentaires préalables à la $11^{\text {ème }}$ proposition: (i) que l'Un contienne le multiple en lui étant supérieur (prop. 1-6); (ii) que la distinction entre le supérieur et l'inférieur, ou l' antérieur et le postérieur, ou encore le prééminent et le subordonné, consiste à attribuer au «meilleur» la plus grande proximité par rapport à l'Un (prop. 7-10), la proximité se mesurant à la possibilité du maintien absolu ou relatif de l'unité avec soi. Les conditions pour que la proposition 11 soit valide et son contenu transparent, consistent donc à faire de la totalité d' une part une unité à l' égard de l' Un, et de l' autre une structure composée de degrés de perfection (en d'autres termes, une structure hiérarchique d'unités, hénades) dont la principale fonction est de se déterminer par distinction radicale de ce qui est le premier (l'Un ou le Bien). Les degrés de perfection des unités se mesurent aux actes de «produire», de «participer» et de «dépendre d'autre que soi» (ne pas être séparé ou autosuffisant), actes qui sont des indicateurs d'infériorité par rapport à l'Un ou au Bien. En tant que totalité la totalité comprend nécessairement tous les étants soumis à ces actes. En revanche, en tant qu'unité la totalité comprend ce qui est supérieur à ces actes («produire», «participer», «dépendre d'autre que soi») et, comme telle, elle transcende nécessairement

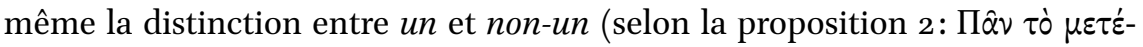

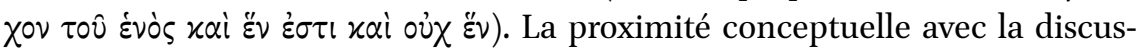
sion du Sophiste $(245 \mathrm{~b}-\mathrm{c})$ sur le rapport entre «tout», «être» et «un» ne fait guère de doute. C' est principalement sur la distinction entre tout-unité et toutensemble de parties que tient la position des monistes face aux pluralistes dans le dialogue entre l'éléate et le platonicien.

La totalité, selon qu' elle est envisagée comme unité ou comme ensemble des étants, semble donc être en mesure de donner la clef de compréhension pour la cause première en tant que précisément cause et non principe; car, à la différence du principe, la cause comporte un aspect actif qui peut entrer en contradiction avec la définition de la totalité, si celle-ci s'identifie à l'Un (immobile et impassible) et non à ce qui est différent de l'Un, en comportant à la fois unité propre et pluralité d' unités distinctes, jusqu'à la singularité de l' individu. Cependant, même si on s'entendait sur la définition bifrons de la totalité, celle- 
ci ne résout pas entièrement le défi du préalable dans l'affirmation que «tout ce qui est procède d'une cause unique et première». L'unicité de la cause ellemême, la primauté et les modalités d'agir dans la situation fixée par la supériorité de ce qui n' agit pas sur ce qui agit, sont les questions qui restent encore en suspens dans ce défi. Essayons dans ce qui suit de les effleurer en analysant la manière dont ces questions surgissent principalement chez trois néoplatoniciens: Victorinus, Saloustios et Proclus. Chez eux, comme chez Plotin et Porphyre que j' évoquerai aussi, la causalité n' est pas réservée au monde physique mais régit aussi le monde intelligible, ayant pour but de définir une relation ontologiquement hétérogène et formellement asymétrique dont seul l'effet peut témoigner puisqu'il est à la fois en dehors et toutefois non-séparé de ce dont il est l' effet. C' est bien cette causalité aporétique néoplatonicienne que Pierre Hadot retrouvait dans la théologie trinitaire de Victorinus et dont il décrivait le fonctionnement en recourant à Plotin, au Porphyre des Sentences et à Proclus.

Il ne s' agit pas de dresser une généalogie de la cause première en comparant les notions et les définitions qui ressortent du fonctionnement décrit par ces auteurs. Il faudrait, à cette fin, remonter à Platon (Timée, Philèbe, Sophiste, Théétète) et à Aristote (Métaphysique Lambda 10), aux débats sur le principe, le tout, l'Un et la nature de la cause dont les néoplatoniciens sont à l'évidence les héritiers tout autant que les critiques. Mon seul et simple but est ici de comparer des textes sans chercher nécessairement les sources. Juxtaposés, les textes des auteurs cités, que beaucoup de fils relient et nombre de positions divergentes séparent irrémédiablement, permettent de cerner par recoupement les lieux spécifiques où la «cause première» intervient dans la structure théorique de l'ensemble en vue de déterminer les ordres, les essences ou les genres premiers. Dans cette entreprise comparative je pars aussi d'un constat: à savoir que la recherche de la cause première est, philosophiquement parlant, non seulement le bien théorique commun de la métaphysique et de la théologie, mais, plus surprenant peut-être, elle est aussi un bien d'ordre pratique dans la mesure où, le premier et l'ultime se rejoignant, la «cause première» se retrouve dans la «cause propre» dont la connaissance par communion et conjonction assure la perfection de l'individu. Or ce bien pratique n'est pas d'emblée propre à une religion. S'il s' est laissé apprivoiser, sa radicalité n'a pourtant jamais pu être entièrement adaptée à des contraintes doctrinales, bien qu' elle ait largement fécondé la réflexion et nourrit l' éthique. C' est cette dernière qui est peut-être le lieu et la fin ultime de la rencontre entre un bien acquis de la métaphysique et une requête de la théologie.

Précisons encore que, ne cherchant pas à reconstruire une généalogie du concept de «cause première», la suite des textes que nous commenterons ne 
respecte pas la chronologie. Elle se présente comme une articulation thématique, faisant travailler ensemble convergences et divergences entre les positions théoriques des cinq philosophes néoplatoniciens qui seront brièvement évoqués au sujet de la cause première et de ce qui en provient.

Cause et précause. La causalité en contexte trinitaire selon Victorinus

Dans la défense antiarienne de la doctrine trinitaire nicéenne, Marius Victorinus s' appuie sur le modèle de la double détermination de l' acte noétique: $m o u$ vement réflexif assurant l'unité formelle de l' acte avec ce qui le produit et avec ce qu'il produit, en même temps qu'actualisation du noûs comme unité substantielle prééminente sur la multiplicité des noêta. Or cette double détermination de l'acte par lequel l' hypostase seconde noue son lien avec l'Un, semble relever d' une interprétation de la causalité première devenue courante dans le sillage de Plotin. Mais que défend le théologien latin en recourant au modèle noétique plotinien? Non seulement la possibilité d'une subsistance de deux natures parfaitement accomplies et parfaitement distinctes dans un seul étant, celui de la seconde hypostase de la Trinité, le Logos incarné, mais aussi la possibilité d'un rapport étroit entre la première et la seconde hypostase, rapport double puisqu' il fait fond sur une «communauté» substantielle et se manifeste comme «acte», en l' occurrence: acte d' engendrement qui simultanément unit substantiellement le Père et le Fils et les différencie par des actions spécifiques. Ce rapport relève donc de l'unité ousiologique (appelée homoousion ou consubstantialité), bien que cette unité soit présentée comme étant concomitante avec la différence, à la fois dans l' acte et dans l' actualisation entéléchique (différence des «opérations», selon le vocabulaire de Victorinus), qui permet de distinguer et de singulariser chacune des trois personnes ou hypostases consubstantielles de la Trinité. Par conséquent, les modalités d'articulation spécifiques au contexte trinitaire entre la consubstantialité des hypostases, l'acte sous-jacent à l'être-existant et les actions propres qui individualisent chaque hypostase, représentent l'un des thèmes de réflexion du théologien latin, dont les références affichées se trouvent évidemment dans le texte évangélique (plus particulièrement chez Jean et chez Paul), tandis que les arguments philosophiques rejoignent la position critique de Plotin envers Aristote, du moins sur la question de l'acte et de la puissance à l'égard des «êtres premiers».

Bien que Victorinus ne puisse pas respecter la séparation radicale, ousiologique et noétique, de l'Un plotinien, car pour lui le Père s'identifie nécessaire- 
ment à l'être, l'usage du modèle plotinien de causalité, de même que l'appel à la réflexivité (ou mouvement immobile) et à la notion opératoire d'image sont frappants dans l'Adversus Arium. Comment procède le théologien? En associant la causalité à la dialectique acte/puissance et à la possibilité conséquente de déterminer des niveaux de réalisation des êtres en fonction d'un ordre de prééminence soit de l' acte soit de la puissance. Or de l'ordre de prééminence que Victorinus établit entre l' acte et la puissance découlent pour lui les distinctions entéléchiques (ou de niveaux de perfection) qui concourent à l' unité divine de la Trinité. Le théologien détermine ces distinctions en fonction de trois critères: (i) le fondement à la fois essentiel (ousiologique) et existentiel (hypostatique); (ii) l'action accomplie à la fois comme mouvement et comme ouvre; enfin (iii) la possibilité différenciée d'intellection qui calque la possibilité spécifique de manifestation.

Réputée pour sa complexité, la démarche du théologien latin s' avère moins compliquée si on tient compte du fait que Victorinus ne cherche pas à produire une démonstration d'école. Son but est de mettre en perspective philosophique les affirmations de la doctrine nicéenne afin de pouvoir répondre à des accusations qui se présentent, elles, comme précisément philosophiques. La dispute avec un vrai ou factice arien appelé Candidus est donc bien une controverse philosophique portant sur l'identité et le statut des «êtres premiers». Les arguments du théologien doivent cependant tenir compte de quelques impératifs de doctrine, dont celui de l'égalité entre les hypostases, ajouté à celui de la consubstantialité, est peut-être le plus contraignant d'une part en raison de son extension à la fois formelle et substantielle (bien que cette extension de l'égalité ne convienne qu' aux substances secondes et non aux premières, selon Aristote $^{2}$ ), et d'autre part en raison de la difficulté à saisir l'égalité quand il s'agit de définir l'ordre d' antériorité de l'acte ou de la puissance, ou lorsqu'il s' agit de saisir la différence des actes tels qu'être et agir, par exemple, actes qui définissent les hypostases individuellement.

Il est donc difficile de faire l'économie d'une compréhension circonstanciée de la manière dont Victorinus applique aux rapports intra-trinitaires la relation acte-puissance en en faisant une relation causale. Pierre Hadot, dans les notes de l'édition du traité de Victorinus ${ }^{3}$, signale l'usage d'un modèle appelé «spécifiquement néoplatonicien» de la causalité, modèle selon lequel la cause demeure immobile en son être tout en étant présente en ses effets, effets qui se convertissent eux-mêmes vers leur cause. On retrouve cette théorie chez

2 Aristote, Métaphysique, $\Delta, 15$, 1021a 9sq. Définissant ce dont la quantité est une, l'égalité concerne les substances qualifiées, secondes.

3 Marius Victorinus, Traités théologiques sur la Trinité, p. 829. 
Plotin (Énnéade IV, viII [6], 6, 10), chez Porphyre (Sentence 24), plus tard chez Proclus (Éléments de théologie 30 $)^{4}$. J'y reviendrai en ajoutant d'autres références à celles évoquées par Hadot. Victorinus se serait servi de cette théorie causale spécifiquement néoplatonicienne pour localiser les passions dans les effets tout en sauvegardant l' impassibilité de l' essence divine dans le Père et le Fils ${ }^{5}$. Il est certain qu'il ne s' agit ni de la distinction entre les deux espèces de causes, «divines» et «par nécessité», établie dans le Timée (68e-69a), ni d'une adaptation réductrice de la théorie aristotélicienne bien connue, car le théologien ne sépare pas les causes par genre mais se contente de distinguer leurs domaines d'application, leurs modalités et leurs effets. Ainsi, la manifestation de la causalité selon Dieu se traduit comme cause de l'être essentiel (ousia), tandis que selon le Logos elle devient cause de subsistance hypostatique, c' est-à-dire détermination de l'être comme existant et donc réduction à la possibilité d'individuation et de différentiation existentielle. Par conséquent, la causalité du Logos est plus restreinte que la causalité divine paternelle puisqu' elle implique la seule «réalité existentielle» (hypostatique) de cet être «second» parmi les «êtres premiers», mais elle est néanmoins identique ou du moins égale à celle du Père puisque ce qui agit comme cause de la subsistence provient toujours du fondement unique et commun de l'ousia qui est cause substantielle (matérielle?) pour l'être. S'ensuit que, selon Victorinus, la cause n'agit qu'en référence à l' acte et à la puissance, qu' elle est donc exclusivement «ontique», jamais formelle, et qu' elle ne sert qu' à déterminer un décalage de «réalité» ou d' «effectivité». Plus exactement, elle sert à déterminer la «réalisation » de l' être en tant que réalité substantielle, qui est une «réalité de puissance» commune partagée par les trois hypostases grâce à l'ousia (divine) commune. Cependant, cette réalité substantielle est inséparable de la réalité subsistentielle (hypostatique ou existentielle), qui constitue une «réalité d' acte» et qui, dans le cas de la Trinité, est unique et propre à chacune des hypostases, à l' exception du Père dont la «réalité existentielle» est subsumée à la «réalité d' acte» du Fils.

Prenons pour exemple un passage du premier livre du traité où est définit ce que serait l' "être» du Père (quod deus est) comme «cause de ce qui est l'être en puissance et cause de tous les existants quant à leur être (esse potentia est,

4 Ces références à Plotin, Porphyre et Proclus sont citées par P. Hadot dans Marius Victorinus, Traités théologiques sur la Trinité. Plus récemment, sur les spécificités néoplatoniciennes de la causalité, voir Chiaradonna 2014.

5 Des passages exemplaires pour cet aspect se trouvent dans les livres I, 13, 11-19 (cité infra, n. 15) et IV, 44, 15-20, des Traités théologiques. 
et omnium quae sunt ad id quod est esse, causa est)» 6 . L' « être » du Père consiste donc à agir en tant que double cause, parce que le Fils, étant cet «être en puissance» dont le Père est la cause, est à son tour, au titre de Fils ou de Logos, cause de la subsistence de l'être, laquelle fait de lui «principe et perfection (principium et perfectio)», donc cause et entéléchie de l' existant. Récurrente, signalée quelquefois sous l'expression causa et praecausa, «cause antérieure » ou «cause de la cause $»^{7}$ pour signifier le propre du Père par rapport au Fils, cette cause dédoublée exprime de toute évidence la difficulté du choix entre la nécessité logique de l'antériorité causale et le respect doctrinal de l'égalité qui suppose aussi la simultanéité et n'admet pas un rapport temporel entre les «êtres premiers» par définition éternels. En même temps, la causalité qui incombe au Logos est elle aussi double, puisque le Logos est cause pour soi et pour les autres, or cette double activité causale sur le plan de la subsistence est précisément la caractéristique des êtres éternels. Nous pouvons voir dans cette tentative de définir le Logos comme cause principale à la fois pour soi et pour

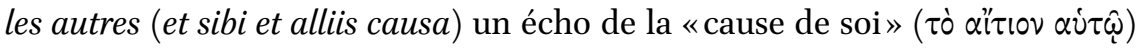
telle qu' elle est évoquée dans De ceternitate mundi par Philon d'Alexandrie (ou Pseudo-Philon, si on n'accepte pas la paternité philonienne de ce traité $)^{8}$. Il

6 Marius Victorinus, Adversus Arium (dans Traités théologiques), I, 34, 8-20: «Toujours est le Père, toujours est le Fils, ils coexistent éternellement.' Ainsi donc, tant que le Fils est toujours consubstantiel, coexistant, et un avec le Père, il est dans le Père (semper consubstantialis, coexsistens, unum exisistens, in patre filius est). Mais lorsqu' il s' actue il procède (cum autem operatur, procedit); et lorsqu'il procède, cette fois c' est le Père qui est dans le Fils. (...) Dieu et le Logos sont un, unis, et à cause de cela consubstantiels. Mais ce qu' est Dieu, en tant qu'être Dieu (sed quod deus, iuxta quod deus est), est cause de ce qui est l'être en puissance et cause de tous les existants quant à leur être (esse potentia est, et omnium quae sunt ad id quod est esse, causa est). Et le Logos, en tant qu'il est Logos, est la puissance issue du Père pour donner subsistence à l'être (paterna est potentia ad subsistere facere ipsum quod est esse), il est luimême l'être originel, et il est principe et fin (principium et perfectio). C'est en effet à partir de cet être universel et de l'être qui est au-dessus de l'universel (esse universale, et supra universale) que l'universalité de l'être, mais, cette fois, répartie selon l'être des genres, l'être des espèces, l'être des individus, reçoit l'être qui est propre à chacun d' eux.» (éd. P. Henry, trad. P. Hadot).

7 Marius Victorinus, Adversus Arium, I, 3, 21-27: «Car seul le Logos, en tant qu' il est Logos pour soi-même et pour les autres, donne l'être à tous les existants (et sibi et aliis ispum quod est esse). Et à cause de cela, il est, sans doute, égal au Père - car la cause principale est cause et pour soi et pour les autres, étant cause par sa puissance et par sa substance (causa enim principalis et sibi et aliis causa est et potentia et substantia causa exsistens) - mais le Père est précause (praecausa autem Pater). D' où il suit que le Fils se distinguera du Père en ce qu'il se meut et se met en acte (quod movetur et operatur), en vue de la manifestation, tandis que le Père, à cause de sa divinité transcendante, agit d'une manière qui nous est inconnaissable.»

8 Philon d'Alexandrie, De ceternitate mundi 70 : «[...] ce qui est pour soi cause d'existence est 
s'agit de l'argument péripatéticien (attribué à Critolaos par l' auteur du traité) en faveur de l'éternité. Or, en réduisant Dieu à l'être, fût-il éternel, le théologien ne peut toutefois pas admettre une procession qui détruirait l'unité substantielle et l'égalité des hypostases, éternelles elles-aussi, de la Trinité. Il cherche alors à re-sémantiser le vocabulaire de la cause en se servant du rapport actepuissance. Quelle qu' elle soit, la cause subordonne l' effet ou alors se disqualifie comme cause et se met elle-même à la place de l'effet. L' argument topique utilisé par le théologien vise à parer à cet inconvénient en situant le Fils dans le Père en tant que puissance de l'être, et le Père dans le Fils (in filio est Pater) selon l'acte ${ }^{9}$. À cette même tâche est assigné le vocabulaire de l'image pour faire de l'acte «l'image de la substance» (substantiae autem dei imago est actio $)^{10}$ et aussi «l'image de ce qui est dans la puissance» (imago est actio, unicuique eorum quae in potentia sunt) $)^{11}$.

On peut considérer que l'usage du vocabulaire de l'image est justifié par l'argument évangélique: le Fils est l'image du Père. Donc ce en quoi l'image est image est la cause de l'image (quoniam autem causa ipsi est id in quo $e s t)^{12}$, puisque si le Père engendre le Fils, c'est au Fils qu'il revient de se

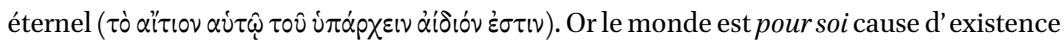

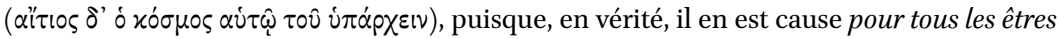

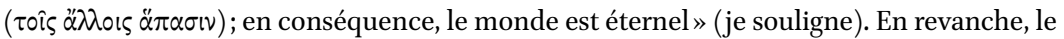
terme praecausa utilisé par Victorinus (passage cité supra, n. 7) serait emprunté au vocabulaire gnostique où il signifiait la «primauté par antériorité»; voir Tardieu 1996, Hadot 1968 et Abramowski 2005 (occ. p. 533).

9 Marius Victorinus, Adversus Arium, I, 34, 8-12 (cité supra n. 6).

10 Marius Victorinus, Adversus Arium, I, 34, 1-6: «Mais l' acte est l'image de la substance de Dieu (substantiae autem dei imago est actio) et il est Fils. [...] Mais parce que ce en quoi elle est image est sa cause (Quoniam autem causa ipsi est id in quo est), l'image elle-même est donc le Fils de ce en quoi elle est (imago ipsa filius est eius in quo est), par une génération ineffable $[\ldots] »$.

11 Marius Victorinus, Adversus Arium, I, 19, 24-29, 35-39: «Cet acte [le Logos], possédant tout ce qui est dans la puissance, le fait sortir au-dehors, selon le mouvement, par la vie et l'intelligence (vita et cognoscentia), et ainsi toutes les choses sont manifestées. C'est pourquoi l' acte est l'image de tout ce qui est dans la puissance (imago est actio, unicuique eorum quae in potentia sunt), spécifiant chacune des choses qui sont dans la puissance et existant par soi-même (speciem perficiens, et exsistens per semet): car aucune substance ne vient du néant (a nihilo enim nulla substantia).[...] parce que ce qui est l'être donne, à l' espèce, ce qui est l'être, et que l'être de l' espèce est l'image de cet être qui, en tant que cause, est le premier être, l'être est donc, pour tout deux, consubstantiel (esse autem spe-

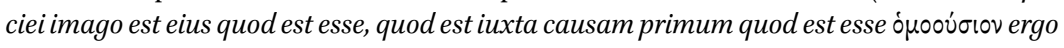
esse ipsis duobus), et le second être est image du premier être (et secundum esse imago est primi esse); je parle de premier et de second, sans considération du temps (sine tempore primum et secundum dico) [...]». 
connaître en tant qu' image du Père. De même dans le passage précédemment cité $^{13}$ où l'action était appelée «image de la puissance» parce que ce en quoi l'acte agit est bien cause de l' action, c' est-à-dire l'ousia paternelle unique sous l'hypostase du Fils. Mais dans les deux cas d' utilisation de l'image pour signifier l'acte et pour montrer la cause (matérielle, formelle ou efficiente), comme dans le cas de l'intériorité et de l'extériorité réciproque du Père et du Fils selon la substance ou selon la subsistence, ce qui est cause de l' acte existentiel, ou de l'action ou de l'agir qui sont propres au Fils, est bien la puissance de l'être qui précède l'acte. Or la puissance ne précède pas l' acte parce que le Père est antérieur au Fils, mais parce que pour Victorinus, comme pour Plotin, la puissance se définit comme ce qui contient la possibilité de l'acte. La puissance se définit donc comme un «acte inné» (intus insitam operationem) $)^{14}$ ou comme un «acte qui n' agit pas» (quod actio inactuosa) ${ }^{15}$, dit Victorinus (comme d'ailleurs Plotin dans Enn. V, vi [24], 6, 4-6), bien que cet «acte inactif» possède toutefois la possibilité d'avancer vers l'agir en touchant ainsi à sa réalisation, à sa fin ou à sa perfection (ab actione procedens in perfectionem veniens $)^{16}$. Cependant, en précédant l'acte, la puissance agit non seulement en n' agissant pas («agit», en l'occurrence, comme possibilité qui lui incombe de manière inconditionnelle), mais produit aussi un bouleversement du statut même de la «réalisation». C'est la possibilité d' agir qui devient ainsi la «réalité» de l'être

13 Cité supra, n. 11.

14 Marius Victorinus, Adversus Arium, I, 4, 1-18: «L'être, c' est le Père, le Logos, l'agir. Et premièrement est l'être (prius est quod est esse), en second lieu, l'agir (secundo quod est operari). À la vérité, l'être lui-même possède à l'intérieur, un acte inné (intus insitam operationem); car sans mouvement, c' est-à-dire, sans acte, quelle possibilité y aurait-il de vie et d'intelligence? [...] l' agir aussi, qui est second, est appelé agir (operari), parce qu'il n' agit pas à l' intérieur, mais à l' extérieur. Car lorsque l' acte se manifeste, c' est alors qu'il est réellement acte et qu' on l'appelle acte (operatio), et c'est alors que l'on conçoit et qu' il y a réellement une autogénération de lui-même (ut generatio sui ipsius et aestimatur et est). Ainsi donc, cela même qui est l' agir a aussi l'être lui-même (ipsum esse habet), ou plutôt il ne l'a pas (magis autem non habet). Car l'agir lui-même est l'être (operari esse $e s t)$ - ils sont en effet ensemble et simplement une seule chose - [...] Et cela qui est l'être en mouvement est la définition de l'être, en tant qu' acte de l'être (quod est esse, secundum actionem)».

15 Marius Victorinus, Adversus Arium, I, 13, 11-19: « [...] il [le Père] est aussi plus grand parce qu'il est acte qui n' agit pas (quod actio inactuosa). [...] Le Fils, par contre, reçoit le don d'être, et s' avançant par l' acte vers l' agir, parvenant à la perfection (in id quod est agere, ab actione procedens in perfectionem veniens), il ne se réalise comme plénitude que dans le mouvement (motu efficitur plenitudo), étant devenu tous les existants. [...] Il est toujours plénitude et toujours réceptacle; et pour cette raison, il est à la fois impassible et passible. Donc à la fois égal et inégal».

16 Ibid. supra. 
premier en assurant la «réalisation» de ce qui s' actualise à partir de celle-ci comme d' une cause première. Ainsi, le recours à l'image, bien que référant aux passages évangéliques où le Fils est appelé image du Père (ou eikôn de Dieu), n' apparaît pas comme un argument en faveur de l'interprétation trinitaire. Tout au contraire, Victorinus semble justifier les relations intra-trinitaires en empruntant l' argument de l' image aux textes philosophiques qui y recourent, en faisant du reflet, de l'image et de la participation iconique les modes spécifiques d'inférence entre la première et la seconde hypostase de la triade plotinienne des principes ou hypostases premières. L' image joue dans ce cas le rôle d'un premier effet de la cause première, effet qui retourne ainsi à la cause le reflet dans lequel la cause peut être saisie et, avec la cause, quelque chose de l'être premier aussi.

Il s' ensuit que la détermination de la réalité ontique de chaque hypostase en tant qu' actualisation de l'être commun, n' est plus assurée par l' acte lui-même (par l'energeia) et par l'accomplissement spécifique (par l'œuvre réalisée), mais bien par une puissance dont la plénitude est intrinsèquement «acte», lequel est simultanément unique et partagé. Cet acte s' identifie à l' être premier en tant qu'être substantiel individuel (ousia) dont les actions ou les œuvres qui singularisent et déterminent l'être individuel ne sont qu' un acte dérivé ou une réalité seconde. On peut rapprocher cette situation, à la fois de distinction et d'inférence, de la théorie plotinienne de l'acte en soi et de l'action ${ }^{17}$. Cette puissance paradoxale, dont la plénitude est acte en soi, demeure néanmoins une puissance selon Victorinus, non un acte, parce qu' elle ne se révèle pas ellemême substantiellement en s' activant perpétuellement dans l'être premier. Le Père ne se révèle comme acte que dans/par le Fils, et non en lui-même: Dieu n' est acte que lorsque le Logos lui-même est (s' actualise). L' acte demeure donc dans la puissance non selon un «avant» et un «après», car l' engendrement du Fils (et c' est valable aussi pour l'Incarnation) n' est que l' acte qui infère, l' acte second, acte qui demeure perpétuellement dans la puissance en tant qu' acte de celle-ci, c' est-à-dire comme image de la puissance (imago est actio, unicuique eorum quae in potentia sunt ${ }^{18}$ ). La puissance apparaît donc comme la réalité fondamentale de l' acte, de la même manière que le modèle n' est pas seulement

17 Il s'agit de la théorie plotinienne dite «des deux actes», appelée ainsi depuis l'article de C. Rutten (Rutten 1956). Considérée tantôt de souche aristotélicienne (C. Rutten), tantôt d'inspiration platonicienne et stoïcienne (Hadot 1968, t. I, p. 228-229), cette théorie est réexposée par E.K. Emilsson (Emilsson 1999; Emilsson 2007, en particulier chap. I, p. 22-42); elle est reprise encore récemment par R. Chiaradonna (Chiaradonna 2014) et A. Michalewski (Michalewski 2014, p. 101-107, 123-128 et 205-207).

18 Marius Victorinus, Adversus Arium, I, 19, 27-28: «image est l' acte de tout ce qui est dans la puissance» (extrait d'un passage cité n. 11), trad. P. Hadot légèrement modifiée. 
la « cause » mais constitue la « réalité» même de l' image, la seule «réalité vraie» de celle-ci qui en est la copie. L'être premier qui recèle ce paradoxal «acte»de la puissance, joue à son tour le rôle de «cause» à la fois par sa puissance et par sa substance; il est donc cause principale et entière d'une «réalité seconde» qui est celle de l' être existentiel (causa enim principalis et sibi et aliis causa est et potentia et substantia causa exsistens $)^{19}$. Ce dernier atteint lui-même sa plénitude lorsqu' il se manifeste comme être existentiel mais, à la différence de l' être premier, il est et se manifeste ainsi lui-même, se montre et opère des actions lui-même, en personne... c' est le cas de le dire. Cette fois-ci la distinction se situe sur le plan de l' action phénoménale, et elle a pour conséquence une nouvelle possibilité de définir la différenciation, possibilité qui est de l'ordre de l'intellection, car l' acte de l'être premier demeure transcendant et inconnaissable (comme dans le cas de la «cause une et première» chez Proclus), alors que l'action existentielle de cet être premier meut et se montre, donc affecte et se laisse saisir dans / par son effet ${ }^{20}$.

Dans ce contexte, l' appel à la « cause » signifie donc non la souscription à un modèle régi par la finalité, mais la nécessité d'inscrire sous un modèle ontologique, et en fin de compte comme un «argument ontologique», l'inversion théologique que Victorinus opère dans le rapport acte-puissance. Cette inversion, réclamée par la doctrine trinitaire, suppose que la puissance ne puisse pas contenir des contradictions à l'égard de la substance mais soit exclusivement ce qui s'actualise toujours, sans limite et sans répit dans le contexte des «réalités véritables», c' est-à-dire des étantités hypostatiques de la Trinité. Mais cette causalité, entièrement fondée surl' acte et la puissance, n' est pas une vraie causalité. Elle n' est ni péripatéticienne ni de type néoplatonicien, car là où chez Plotin, en contexte noétique, l'acte comporte une puissance puisqu'il peut agir comme «acte essentiel» et s'identifier ainsi à toutes les choses existentielles qui lui sont internes, en les accomplissant lorsqu' il s' effectue lui-même

19 Passage cité supra n. 7.

20 Victorinus revient sur cette unité d'acte, de puissance et de substance, ainsi que sur la distinction dialectique par le plan de réalisation de l'acte dans le troisième livre du traité lorsqu'il définit ainsi la Trinité: «[...] les trois sont un, par leur puissance et substance. Pourtant les deux premiers sont un, tout en étant différents en ce que le Père est existence qui a valeur d'acte, c' est-à-dire substantialité (actualis exsistentia, id est substantialitas), tandis que le Fils est acte qui a valeur d' existence (actus exsistentialis). Les deux autres, par contre, sont deux, de telle sorte que le Christ et l'Esprit-Saint sont deux en un, c' està-dire en un seul mouvement (in motu), et ainsi ils sont deux, comme une unité peut être deux (ut unum duo). Les deux premiers, par contre, sont comme un 'deux' qui est un (ut duo unum). Ainsi étant à la fois 'deux en un' et 'deux un', la trinité est Un (trinitas exsistit unum)». (Adversus Arium, III, 18, 13-18). Voir aussi infra, n. 33 . 
en tant qu' intellection séparée, chez Victorinus, à l' inverse, c' est la puissance qui contient l'acte en premier; en l'occurrence, c' est la puissance du Père qui contient l'acte actué comme «être premier» et qui s' accomplit existentiellement dans l'hypostase du Fils ou du Logos, en assurant aussi la possibilité de son intellection et en donnant donc une connaissance divine de lui-même à travers celui qu'il n' est pas.

\section{Principe ou cause première selon le modèle néoplatonicien de causalité. Plotin, Porphyre, Saloustios}

Revenons à l'hypothèse concernant l'usage par Victorinus d'une causalité de type néoplatonicien, mais élargissons maintenant le cadre des références citées par Hadot afin d'en définir les ressorts. Il faudrait notamment ajouter les propositions et gloses 26 et 35 des Éléments de théologie encore plus éclairants que la proposition 30 mentionnée par Hadot sur la persistance de la cause en elle-même sans déplacement ni amoindrissement, et sur la conversion des effets vers leur cause. Mais, avant d'entrer dans l'univers conceptuel des Éléments, signalons quelques passages de Plotin et de Porphyre, et arrêtons-nous brièvement sur un écrit de Saloustios, en remarquant d'emblée que chez Porphyre par exemple, comme chez Proclus plus tard, cette causalité dite «néoplatonicienne» n' est pas exprimée en termes d'engendrement et de naissance mais se définit en termes de procession tant qu' elle concerne la sphère des incorporels. Il en est de même chez Plotin, bien que ce dernier utilise aussi le terme de «semence» pour désigner le principe supérieur ou premier comme «cause» dont proviendrait le sensible, ce dernier se déployant comme multiple à partir d'un principe qui demeure en lui-même indivisible (Enn. IV, 8 [6], 6, 1-9 $)^{21}$. Il s' ensuit que le rapport cause-effet n' est pas symétrique chez Plotin comme il tend à l'être chez Victorinus, dès lors que Plotin

21 Plotin, Ennéades IV, 8 [6], 6, 1-9: «Si donc il est nécessaire qu'il n’y ait pas qu'une seule chose - car alors toutes choses resteraient cachées puisqu' elles n' ont pas de forme dans l'Un, aucun être n' existerait, car l'Un resterait en lui-même, et il n'y aurait pas de pluralité de ces êtres nés de l'Un, de sorte que n' existerait pas après eux la procession des êtres qui ont reçu le rang d'âmes - de la même manière, il ne faut pas qu' existent seulement des âmes, sans que les objets qu' elles font naître ne deviennent visibles, s' il est vrai qu' en chacune existe par nature la capacité de produire ce qui est après elle et de se développer en allant, comme une semence, d' un principe indivisible vers un état final qui est l' objet sensible». Sur la modalité dont Plotin envisage le rôle causal de l'Un (entre la cause efficiente et la cause finale) à l'égard de l'hypostase seconde, l'Intellect (noûs), voir en particulier Bussanich 1996, ainsi que D’Ancona 1996. 
étend la procession et la production ou l'engendrement à l' ensemble des êtres, comprenant les âmes et les choses sensibles (corruptibles), tandis que l' analyse du théologien concerne uniquement les hypostases de la Trinité. Remarquons en outre que la causalité dite «néoplatonicienne » est décrite selon le procès de division et selon la manifestation comme advenue au visible, mais que Plotin ne l'associe pas à la dialectique acte-puissance et qu'il installe, au contraire, une vision hiérarchique des réalités entre celles qui sont propres à la cause et celles qui constituent l'effet. Or la distinction d'ordre ontique (substance, espèce, individu) dont se sert Victorinus pour définir la Trinité, ne comporte (et pour cause) ni division ni hiérarchie comme chez Plotin, malgré la prééminence du Père affirmée en termes de primauté de la puissance sur l'acte.

Pour mieux cerner les rapprochements et les différences entre ces deux usages, la Sentence 24 de Porphyre ${ }^{22}$ pourrait s' avérer éclairante. On voit se dessiner la ligne de fracture qui sépare la procession néoplatonicienne à partir d'un principe premier, décrite ici de manière claire et concise, de la conception chrétienne qui emprunte structure et concepts aux néoplatoniciens mais s'en sert pour défendre une théorie ontologique différente, fondée sur la prééminence de l'être dans son identité avec l'Un. Pour Porphyre, la procession des incorruptibles établit une hiérarchie dans la subsistance au sein même de la classe des incorporels, tandis que pour Victorinus la distinction entre les identités hypostatiques dans la Trinité s' établit par des actes spécifiques (genèse ou naissance et procession), or ces identités, qui sont elles-mêmes incorruptibles, entretiennent un rapport de causalité réciproque en raison même des actes (ou opérations) qui les déterminent et les spécialisent (engendrement, naissance, procession), mais ne sont pas «moindre» sur le plan de la subsistence que le principe unique du Père. L'égalité subsistentielle (hypostatique) représente le fondement même de la Trinité; chez des théologiens grecs contemporains de Victorinus, comme Basile de Césarée, elle est en outre doublée par une égalité d'ordre doxologique qui renforce le caractère subsistentiel par sa reconnaissance dans la manifestation ${ }^{23}$. La procession porphyrienne, comme

22 Porphyre, Sentence 24, p. 325: «Dans le cas des vies incorporelles, les processions ( $\pi$ póo-

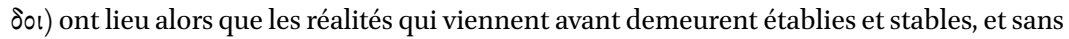
qu' elles ne corrompent rien d'elles-mêmes pour faire subsister les êtres inférieurs à elles

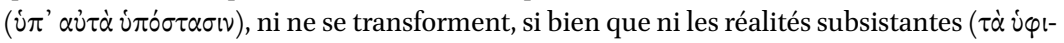
$\sigma \tau \dot{\alpha} \mu \varepsilon v \alpha)$ ne subsistent ( $0 \dot{\delta} \delta \dot{\varepsilon} . .$. í $\varphi i \sigma \tau \alpha \tau \alpha l)$ avec quelque corruption ou changement [...], ni ne naissent ( ment. Ces réalités ne sont donc soumises ni à la naissance ni à la corruption et, de ce point de vue, elles sont venues à l' être sans naissance ( $\alpha \gamma \varepsilon v \eta \dot{\tau} \tau \omega \varsigma)$ et sans corruption».

23 Je me permets de renvoyer à un article consacré à cette question et à une comparaison entre ces deux théologiens de la Trinité, Vasiliu 2014a. 
d' ailleurs celle décrite par Plotin, impose en revanche une vision hiérarchique, qui subordonne l'être à l'Un, et situe les êtres qui procèdent sur une échelle de perfections dans leur subsistence même, bien qu' il s' agisse d' êtres incorporels et parfaits en eux-mêmes.

Décrivant l' advenue à la subsistance des incorporels, la Sentence 24 ne présente pas la procession des réalités inférieures à partir des «êtres supérieurs» comme l'effet nécessaire d'un rapport causal. Pourquoi Porphyre évite-t-il de parler de cause dans ce contexte, alors que Victorinus s' en sert, même s'il est visiblement embarrassé et obligé d' inventer une «précause» pour différencier les causalités spécifiques du Père et du Fils? Pour Porphyre, les supérieurs, éternellement incorporels et incorruptibles, sont supérieurs aux inférieurs qui en proviennent sans agir et sans subir non plus de corruption ou de transformation. À leur tour, ceux qui en proviennent sont exempts de toute corruption, changement ou naissance, tant que les processions ( proodoi) concernent le cas des incorporels. La conclusion de la Sentence 14 va dans le même sens, tout en précisant comment il faut entendre le rapport entre être causé et naître dans le cas des incorporels ${ }^{24}$, tandis que dans la Sentence 44 Porphyre évoque, à l' aide d'une métaphore, le mouvement de reflux qui illustre la conception spécifiquement néoplatonicienne du rapport causal, l' effet retournant à la cause comme un flot à sa source, se convertissant donc au lieu de se déverser, et témoignant ainsi de la cause dont il est l' effet ${ }^{25}$. Bien que Porphyre fasse à chaque fois référence à un cas particulier (les incorporels, les incomposés, l'âme), la situation évoquée reproduit toujours le même schéma univoque selon lequel le principe n' est cause que par retour de ce dont il est cause comme malgré lui, sans qu' il ait été affecté par son actualisation comme cause, ou par une perfection qui se serait accomplie dans l' effet produit. Victorinus, en revanche, définit la distinction des identités subsistantes dans la Trinité précisément en termes de rapport

24 Porphyre, Sentence 14, 10-13, p. 315: «... les uns naissent et son soumis à la dissolution et à la destruction, tandis que les autres, au sens où ils sont incomposés et pour cette raison à la fois indissolubles et indestructibles, ne naissent pas, mais au sens où ils dépendent

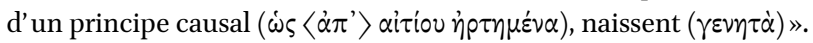

25 Porphyre, Sentence 44, 42-43: «car elle [l'âme] ressemble à une source qui ne se déverse

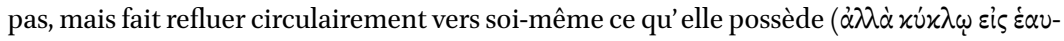

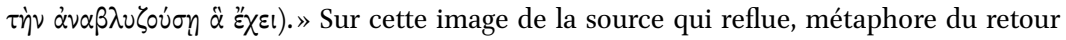
de l'âme et souvenir du Styx qui semble remonter à Hésiode (Travaux, 595), dont on retrouve encore un écho chez Denys l'Aréopagite (Epist., IX, 1), voir J. Pépin et R. Goulet dans Porphyre, Sentences, t. II, p. 776-777. Notons toutefois que les commentaires cités ne font pas de rapprochement entre ce mouvement de retour vers la source et le rapport cause-effet caractéristique dans la définition du mouvement de la procession des incorporels dans le néoplatonisme, ce que P. Hadot appelle la spécificité de la causalité néoplatonicienne, à savoir la détermination de la cause par l' effet. 
de causalité à partir du Père, en introduisant nécessairement une génération, voire une naissance, même si celle-ci se produit sans corruption, et en établissant en même temps une symétrie et une réciprocité dans la fonction de cause entre le Père et le Fils: le premier est cause du second sur le plan substantiel, le second est cause du premier sur le plan phénoménal. La réciprocité et une certaine symétrie dans le rôle causal sont évidemment réclamées par l'égalité stricte entre les hypostases stipulée par la doctrine nicéenne, bien que chacun des deux est en réalité cause d' autre chose.

Mais, les écarts spécifiquement chrétiens mis à part, la procession peut-elle être considérée comme une relation de type causal dans le cas des «incorruptibles»? Ou, plus précisément, peut-on appliquer le vocabulaire de la causalité pour définir ce que les néoplatoniciens tentent de saisir en parlant de procession à partir de ce qui est premier en tant que «principe», «être», «essence», «puissance» ou «cause» notamment? Voyons comment de ce qui est appelée «cause première » (prôtê aitia) procède quelque chose qui, selon la théorie néoplatonicienne, ne peut pas se séparer de cette cause, et comment cette cause agit non pas sans agir, à la manière de la «précause» chez Victorinus ou de l'acte dirigé vers le Bien chez Plotin (Enn. v, 6 [24], 6, 4-6), mais agit sans que son état d'impassibilité soit affecté par le rôle de cause et sans que l' effet puisse ou doive s' en séparer.

Avant d'analyser quelques passages des Éléments de théologie de Proclus qui éclairent le mieux, tel que le suggère Hadot, l' usage spécifique de la causalité en contexte néoplatonicien, arrêtons-nous un instant sur un autre néoplatonicien, Saloustios, le théologien proche de Julien, qui se situe, lui, dans le sillage

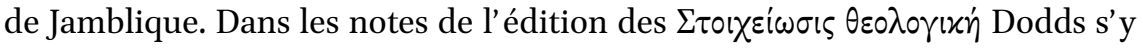
réfère plusieurs fois, comme pour mettre davantage en évidence le penchant jamblichéen des Éléments. L'auteur du traité Des dieux et du monde pourrait en effet nous aider à cerner certains lieux où se dessine le clivage théorique entre Plotin, Porphyre, Victorinus d'une part, Proclus et la postérité, de l' autre, au sujet de la cause première et de ce qui en provient, ainsi que de la manière dont l' effet intrinsèque à la cause révèle la cause en y demeurant ${ }^{26}$.

Pour Saloustios, à la «cause première» répond la «puissance première»

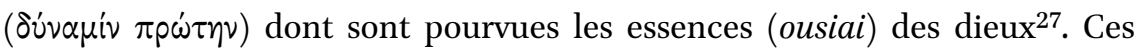

26 Dans Vasiliu 2014b nous avons déjà évoqué les principaux repères textuels dont nous reprenons et développons ici l' analyse, en insistant sur les traits particuliers de cette causalité première, à la fois proche et distincte de la causalité de soi, propre aux principes ou aux essences divines.

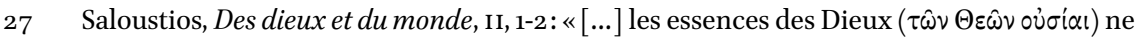

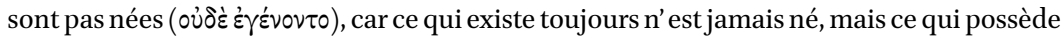


essences ne proviennent pas des corps, sont impassibles et éternelles car elles ne sont pas nées, et comportent une puissance qui est, elle aussi, incorporelle comme toutes les puissances. Mais le propre de cette «puissance première» dont sont pourvues les ousiai des dieux vient de l'état de non-séparation dans lequel se trouvent ces ousiai à la fois entre elles et à l'égard de la «cause première». Les ousiai divines ne sont pas séparées entre elles bien qu' elles soient multiples, et se tiennent toutes dans la «cause première» dont elles ne sont pas non plus séparées. La comparaison avec la non-séparation entre les pensées et l'intellect permet de mieux saisir le propos elliptique de Saloustios. En raison de la non-séparation, la multiplicité des ousiai considérée comme une totalité constitue de facto une unité. Cette unité se définit comme «puissance première» de la même manière que les pensées multiples se trouvent toutes réunies dans l'unité de l' intellect par la puissance commune de l' intellection. Or cette «puissance première» commune à l' unité des ousiai n' est elle-même pas séparée de la «cause première» dont elle est en quelque sorte l' effet. En revenant à l' exemple des pensées et du noûs: l'ensemble des pensées partage la puissance commune de l'intellection qui n'est pas séparée de la «cause première», laquelle s'identifie à son tour au noûs lui-même. Mais l'emploi du terme «puissance» (dunamis) comporte une équivocité, exploitée ici de manière sans doute volontaire, en indiquant un sens d'emblée actif à l'égard des ousiai impassibles et éternelles, et un sens passif qui convient tout autant au statut d' effet de la «cause première » qu' au statut impassible de la «cause première» elle-même. Cette «puissance première» est donc l'effet immanent qui demeure dans la «cause première» et qui se convertit en permanence à/vers celle-ci.

Quelques chapitres plus loin, Saloustios ajoute certains traits qui permettent de mieux saisir la «cause première » dans son rapport à la dunamis et à l'ousia. Le chapitre $v$, le premier de la véritable entrée en matière du traité, y est entièrement consacré. L' auteur esquisse une définition de la «cause première » et de ce qui s' en suit, à savoir les ordres ou les classes des Dieux $\left(\tau \hat{\omega} \nu \Theta \varepsilon \hat{\omega} \nu \tau \alpha^{\prime} \xi \varepsilon ı \zeta\right)^{28}$.

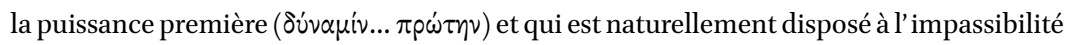
existe toujours. [...] Elles ne sont pas [formées] à partir des corps, car les puissances des

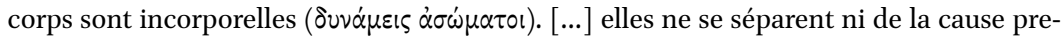

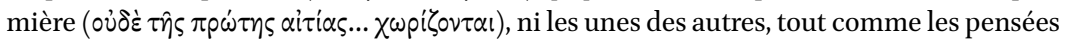

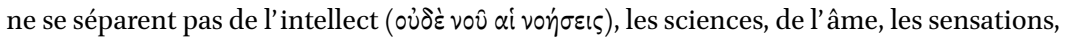
du vivant».

28 Saloustios, Des dieux et du monde, v, 2-3: «2. La cause première se doit d'être une (Tìv

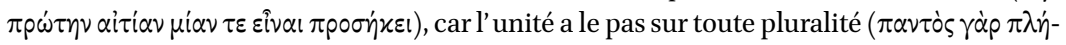

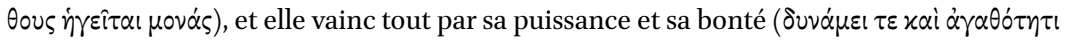
$\pi \dot{\alpha} \nu \tau \alpha \nu i x \hat{\alpha})$; c' est même pourquoi tout doit nécessairement y participer ( $\pi \dot{\alpha} \nu \tau \alpha \mu \varepsilon \tau \varepsilon \dot{\chi} \chi \varepsilon เ \nu)$, 
La «cause première» est unique et a la puissance sur tout. De cette puissance «tout participe» $\left(\pi \alpha^{\prime} \nu \tau \alpha \mu \varepsilon \tau \varepsilon \dot{\chi} \chi \varepsilon \nu\right)$, y compris la «cause première» qui ne peut pas s'en détacher. L'unicité d'une part, l'unité avec la puissance et avec le tout d'autre part, constituent donc les deux déterminants de la «cause première ». Mais comment faut-il comprendre l' unité de la cause avec la puissance et implicitement avec le tout? Il ne faut pas l' entendre comme une participation ( $\mu \varepsilon \tau \varepsilon \dot{\chi} \chi v(\nu)$ de la part de la «cause première», même si la cause ne peut pas s'abstraire $(\dot{\alpha} \varphi \dot{\varepsilon} \xi \varepsilon l)$ de la puissance, dit Saloustios, en raison de sa bonté ( $\dot{\alpha} \gamma \alpha-$ $\theta o ́ \tau \eta \tau \alpha)$. La participation définit ce qui relie le « tout» à la «puissance» comme l' animé à l'âme ou l' intellectif à l' intellect. En revanche, le «tout» ne participe pas de la «cause première», car celle-ci, tel un «principe» ou un «premier» ( $\tau$ ò

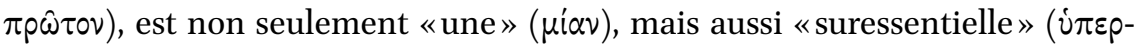
ov́б(ov). La cause a en elle la puissance dont participe le «tout», et l'unité qui relie cause et puissance est bien de l'ordre de la participation de la part de la puissance que recèle la cause, mais elle ne l' est pas de la part de la cause qui possède la puissance et n'a donc pas besoin d'y participer. Car la cause étant une, elle ne se divise pas et ne se sépare pas d'elle-même, et si elle possède de facto la puissance, elle la possède comme un effet de sa propre «nature», la bonté, qui l' empêche de s' en écarter et de s'écarter ainsi de tout ce qui participe de sa puissance ${ }^{29}$. Cependant, n'étant pas une ousia mais étant suressentielle

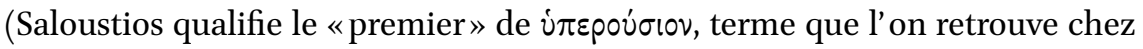
Proclus), la cause n'est pas connaissable autrement que par son unicité, par la puissance (elle-même indicible, apporêta, et dont tout participe) et par sa position qui la situe en amont des ousiai premières qui sont les Dieux. À leur tour, les ousiai divines, du fait de participer en premier de la puissance pre-

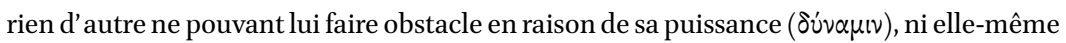
s' écarter $(\dot{\alpha} \varphi \varepsilon \xi \xi \varepsilon \iota)$ en raison de sa bonté.» «3. [...] le [principe] premier doit être nécessai-

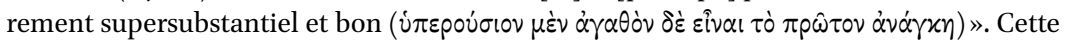
affirmation est formulée à la fin du raisonnement par analogie avec la participation, raisonnement à travers lequel Saloustios cherche à cerner la «cause première»: si elle était âme $\left(\psi v \chi \eta^{\prime}\right)$, tout serait animé ( $\left.\varepsilon^{\prime \prime} \mu \psi \chi \chi \alpha\right)$, si elle était intellect (vov̂), tout serait intellectuel (vospó) etc. Or le «premier [principe]» comme suressentiel correspond au dernier cas évoqué, celui qui nie la réduction de la «cause première» à une essence et à l'être. Il s' ensuit que la «cause première » n' est ni âme, ni intellect, ni essence, ni être premier, mais

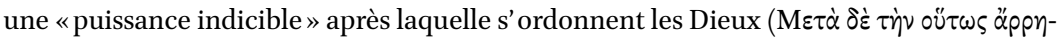

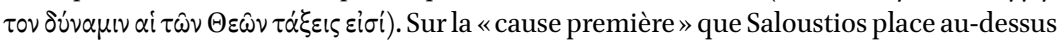
de l' essence, voir Trambrun-Krasker 2006, p. 133 (en rapport avec les écrits de l'empereur Julien et du rhéteur Libanios).

29 C'est le schéma asymétrique propre à la participation platonicienne, décrit par Ferrari 2007, p. 147-172; Ferrari 2010; Ferrari 2015, p. 219-232. 
mière, se tiennent elles-aussi dans la «cause première», ou, plus précisément, n' en sont pas séparées, comme l' avait affirmé Saloustios dans le chapitre II de son traité théologique ${ }^{30}$.

On ne s'attardera pas sur la subtile distinction lexicale entre l'impossibilité de la «cause première» de s' éloigner d' elle-même $(\dot{\alpha} \pi-\varepsilon \chi \chi \omega)$ et de se tenir ainsi à l'écart de la puissance dont tout participe, et l'impossibilité des ousiai de se séparer ou de se diviser $\left(\chi \omega\right.$ pi $\left._{\zeta} \omega\right)$ aussi bien entre elles qu'à l'égard de la «cause première», le multiple se tenant ainsi à l'intérieur de l' unité aussi bien sur le plan des essences premières (ou divines) que sur le plan des intelligibles. On retiendra cependant l'absence chez Saloustios à la fois du vocabulaire de l'acte et de la procession, au profit d'un lexique privilégiant l'unité, l'union, la communion, la non-séparation et la non-division. Or ce choix est un indice transparent de l'orientation du traité: connaître les dieux permet de mieux participer aux cultes en atteignant la félicité ( $\varepsilon \dot{\delta} \delta \alpha \mu o v i \alpha)$ qui corres-

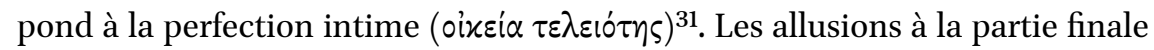
du Timée (89d6-9od7) sont difficiles à manquer. Soigner, tel un «dieu» intime, le daimôn avec lequel cohabite la partie supérieure de l'âme et pratiquer les sciences qui permettent à la pensée de suivre le mouvement parfait des astres et de connaitre l'harmonie du monde, sont les deux perfections qui rendent l'homme heureux, semblable aux dieux. Les échos plotiniens sont eux aussi présents et renvoient à de nombreux contextes. Évoquons un seul, un passage de l'Ennéade v, 3, 49, 12, 40-46, plus particulièrement frappant en raison de cette proximité thématique révélée par la non-séparation de l' acte et de l' activité qui en découle; Plotin y décrit ainsi le rapport entre intellection et connaissance. Mais, pour le platonicien de l'époque de Julien la réflexion autour de cette intellection première et du bonheur de cette pratique conduit vers la communion, non vers l'intellection elle-même. La perfection n'est pas ici l' entéléchie des facultés propres; la teleiotês vise l'union avec la perfection de la cause, or cette union se traduit par l'intégration dans son intimité de la communion de cha-

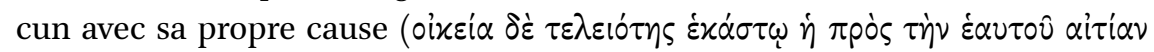

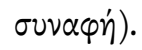

Rejoignant sa propre cause et la touchant presque, la singularité s' autotranscende puisqu' elle participe ainsi du tout, qui participe à son tour de la puissance, laquelle n' est pas séparée des ousiai des dieux, elles-mêmes non-

30 Saloustios, Des dieux et du monde, II, 2, cité supra n. 27.

31 Saloustios, Des dieux et du monde, XVI, 1: «[...] le bonheur de toute chose est son intime

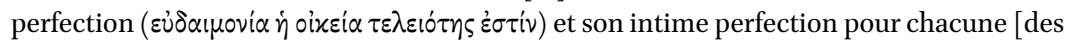

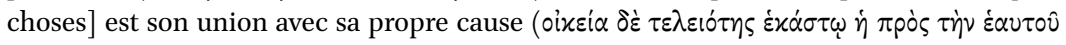

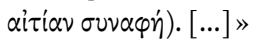


séparées de la «cause première». Outre l' enchaînement qui se déploie à partir du télescopage de la cause immanente et de la perfection, l'emploi du terme

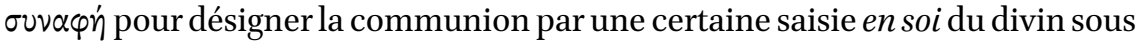
le mode d'une appréhension de la «cause première» dans sa propre cause, est lui aussi symptomatique à la fois pour l'héritage et pour la postérité de cet abrégé de théologie et de mystique néoplatonicienne. L'idée de réaliser la perfection de chaque identité en touchant dans son intimité à sa propre cause, reprend thématiquement le principe aristotélicien de l'identité qui, se situant à l' égard d' elle-même, permet de passer de la simplicité absolue de l'unique à une dualité du «même», dualité résultant du fait de s'y rapporter: lorsqu' on dit

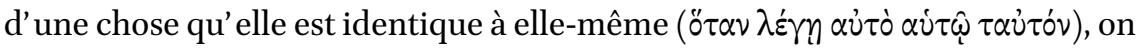

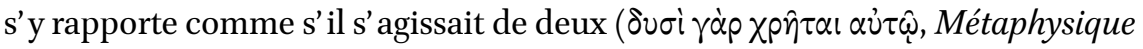
$\Delta$, 1018a9). Sur cette définition de l'identité impliquant de manière référentielle une dualité à l'égard d' elle-même, la réflexion médio-platonicienne, de même que plus tard la pensée trinitaire chrétienne, ont essayé de construire la relation de dédoublement de la substance unique qui s' autoconstitue en comportant une idée d' autre chose que soi ou en connaissant sa propre cause comme cause et perfection de tout. Rappelons à cet égard un passage du fragment 16 de Numénius: «le Second [i.e. le Démiurge de l' essence] qui est double, pro-

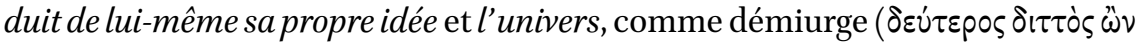

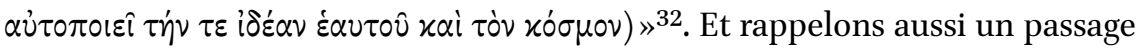
particulièrement éclairant et explicite du traité trinitaire de Victorinus, l'un des nombreux passages à même d'illustrer ce même processus, à cette différence près que le théologien romain traduit toujours en termes d'acte et d'actualité le rapport de dédoublement de la substance unique et simple qui naît de soi et existe par soi comme si elle était autre que soi (sed simplicitate ex se atque in se exsistentis, quasi alterius substantiae duplicatum $)^{33}$.

32 Numénius, Fragments, v, fr. 16, 10-11.

33 Marius Victorinus, Adversus Arium, IV, 10, 3-15: «Il vit, dis-je, et dans l' acte de vivre, dans l' exercice même de l'acte de vivre (in actu vivendi et ipso opere), en vivant, il actue la vie (cum vivit, operatur). [...] Et parce que le 'Il vit' lui-même est substance (ipsum substantia est), ce qui provient de ce 'Il vit' (quod est ab eo quod vivit), la vie elle-même est substance, substance pareille, substance identique, substance égale et coexistante (vita ipsa substantia est, par, eadem, aequalis ac simul), puisque le 'Il vit' est lui-même vie, et que la vie elle-même se pose en 'vivant', afin d'être la vie. [...] Il s' implique mutuellement, en chacun est aussi l' autre, non pas comme quelque chose qui viendrait redoubler le premier et s' ajouter à lui (non ut geminum et adiectum), mais ils sont dédoublés en la simplicité même d' une unique substance qui naît de soi et est en soi, comme si elle était autre que soi (sed simplicitate ex se atque in se exsistentis, quasi alterius substantiae duplicatum)». La définition même de la Trinité, en III, 18, 13-18, suit ce même modèle dialectique posant unicité et coexistence de chacune des hypostases premières dans l' autre, à travers une existence qui 
Quant à l'usage du terme sunaphê, celui-ci indique le registre spécifiquement religieux dans lequel s' inscrit le traité de Saloustios. Si en contexte chrétien Basile de Césarée, par exemple, emploie ce terme dans le Traitésur l'EspritSaint pour désigner l' union sans mélange des hupostaseis divines sous la modalité d'une réflexivité réciproque qui induit l'unité de la connaissance que le divin a de lui-même, connaissance assurée par l'unité essentielle des hypostases et non divisée selon les actes spécifiques, chez Proclus, en revanche, sunapheia désigne une forme de connaissance immanente de la divinité par l'initié dans le cadre d'un rituel. Cette connaissance, que l'on qualifierait d'expression de la foi, s'apparente à une sorte d' adhésion qui va avec la communion (koinônia) et qui, conséquente à l' epistrophê, à la conversion, se manifeste par la ressemblance (homoiôsis). Les occurrences de ce terme se retrouvent aussi bien dans les Éléments (dans le commentaire de la proposition 32, par exemple $)^{34}$, que dans la Théologie platonicienne ${ }^{35}$, Proclus prenant quelque fois le soin de préciser le mode de connaissance entendu par cette proximité, voire ce «contact» incorporel. Saloustios se sert, lui, de ce terme comme pour donner à la cause propre une dimension immanente à l'individu et assurer ainsi une relation directe, malgré l'abstraction inhérente au lexique des principes, de la cause première et des essences des Dieux. Didactique et synthétique, le traité de Saloustios a tout d'un manuel. Il n'est cependant pas un abrégé de théologie adressé à des philosophes, mais une introduction à la pratique éclairée de cette nouvelle religion appelée «platonicienne».

a valeur d' acte, c' est-à-dire s' actualise en chacune, et qui est par conséquent soit substantialité (id est substantialitas), soit existentialité (actus exsistentialis). Ce dernier passage est cité supra n. 20. Pour la perfection qui consiste à s' accomplir en tout, voir aussi Adversus Arium, I, 13, 11-19, passage cité supra $\mathrm{n} .15$.

34 Dans le commentaire de la proposition $3^{2}$ des Éléments de théologie consacrée à l'epistrophê et la homoiôsis engendrée par la conversion et devenue, comme par retour, expression de la perfection de la conversion elle-même, Proclus utilise deux fois le terme sunaphê en conjonction avec la communion (koinônia). Ailleurs, il l' associe aussi avec la parenté naturelle (sungenia, sumphuês), la contiguïté (sunêptai), la proximité, la sympathie (sumpaskhein). Voici le passage évoqué des Éléments: «If, then, reversion is a commu-

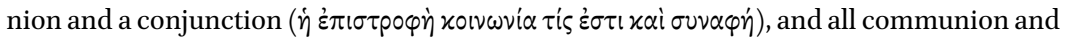

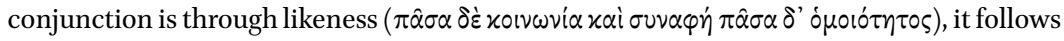

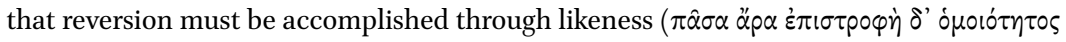
$\dot{\alpha} \pi \circ \tau \varepsilon \lambda \circ i ̂ \tau \circ \alpha \dot{\alpha} v) »$.

35 Proclus explicite dans ce cadre l'usage du terme: «c'est pourquoi... les théologiens

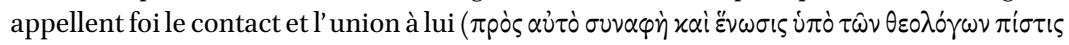
$\dot{\alpha} \pi \circ x \alpha \lambda \varepsilon i \tau \alpha l)$ [il s' agit du bien]; et pas seulement les théologiens, mais s'il faut dire notre

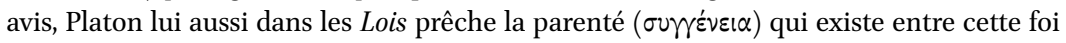
d'une part, la vérité et l'amour d' autre part». (Proclus, Théologie platonicienne, I, 25, I.1, p. 112). Les occurrences de sunaphê chez Basile sont très nombreuses dans le traité cité. 

première selon Proclus, ou le paradoxe du retour systématique

Dans les Éléments de théologie Proclus thématise la «cause première» en fixant ainsi les traits caractéristiques de la causalité dite «néoplatonicienne». Le paradoxe que recèle la «cause première» tient à son effet : l' effet de cette cause est tel que non seulement il ne se sépare pas de la cause, mais à peine s'en distingue-t-il qu'il revient aussitôt à la cause et n' a de cesse de la rejoindre et de s'y identifier. De surcroît, non seulement tout participe de cette cause, mais le fait même d'y participer assure aux participants, à tous comme à chacun, la perfection. La « cause première» agit-elle alors comme une cause, ou mieux vaut-il parler, comme Porphyre, de procession immobile d' êtres ou d' essences impassibles à partir d'un principe, ou bien, comme Plotin et Victorinus, d'un acte premier qui n' agit pas? Faut-il, autrement dit, abandonner le terme de « cause» pour ce qui est du «premier» en considérant que la cause est ici synonyme de «principe » et qu' elle ne remplit nullement la fonction d' une cause? On court cependant le risque de rester enfermés dans la tautologie du principe-origine et de faire remonter par conséquent ce principe «premier» vers un «principe des principes », régression à l'infini dont le danger était signalé par Aristote ( $\alpha \lambda \lambda^{\prime}$

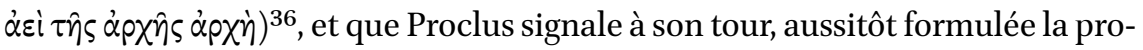
position 11 au sujet de la totalité qui provient de la cause unique première ${ }^{37}$. C' est la proposition citée au début de l'étude, à laquelle j' ajoute maintenant un résumé du commentaire proclien. Une «cause première» peut-elle cacher derrière elle une autre «cause première», dont elle-même proviendrait? Une telle situation empêcherait la connaissance des choses, or ne pas connaître l'existant est impossible, tout comme sont impossibles l'absence de cause, l'échange de place entre la cause et le causé ou encore la régression infinie.

36 Aristote, Métaphysique, $\Lambda$, 1075b26-27: «Si d' ailleurs l'on veut qu'il n'y ait pas d'autres êtres en dehors des êtres sensibles, il n'y aura ni premier principe, ni ordre, ni génération, ni mouvements célestes, mais il y aura principe de principe à l'infini ( $\alpha \lambda \lambda^{\prime} \dot{\alpha} \varepsilon \dot{\imath} \tau \hat{\eta} \varsigma \grave{\alpha} p X \hat{\eta} \varsigma$

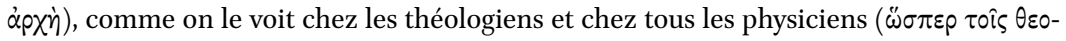

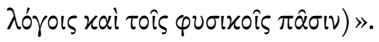

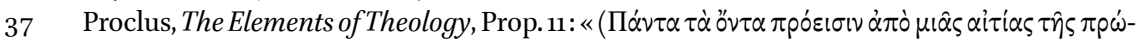
$\tau \eta \varsigma)$. All that exists proceeds from a single first cause. For otherwise all things were uncaused

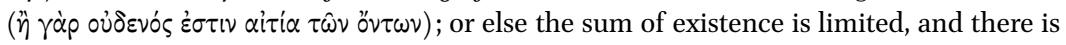

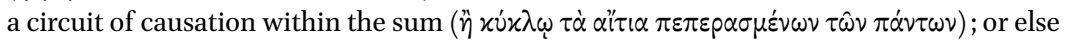
there will be regress to infinity, cause lying behind cause, so that the position of prior

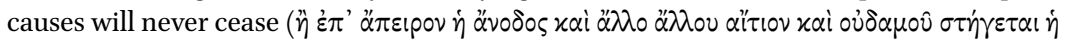
$\tau \hat{\jmath} \varsigma \alpha i \tau i \alpha \varsigma \pi p \circ \ddot{\pi} \pi \delta ́ \sigma \alpha \sigma \iota \varsigma) »$. 
Une «cause première » est par conséquent nécessaire à tous les étants, affirme Proclus, de même qu' un premier principe a été établi, à savoir que le multiple tout entier est postérieur à l' $U n^{38}$.

Proclus énumère ainsi les arguments qui le conduisent à affirmer par déduction la nécessité de la cause première: les choses n'étant pas sans cause, ce qui est causé étant distinct de la cause et la remonté à l'infini étant une impasse, il ne reste plus qu'à admettre l'existence d'une cause première de toutes les choses existantes. Mais cette assertion entraine une autre, à savoir que le multiple provient alors de l' Un, comme les branches à partir d' une racine. Proclus admet cependant, et ce dès la proposition suivante, que la «cause première» de tous les étants, de même que le «principe» de tous les étants, s' identifie(nt)

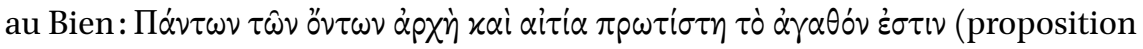
$12)^{39}$. Le dédoublement entre «cause première» et «principe» s' impose donc, mais non sans soulever des questions. Le danger serait de les confondre, ou alors de considérer qu'il y aurait deux causes premières. Mais Proclus rappelle la proposition précédente: il n'y a qu'une seule ( $\mathrm{mia}$ ) cause première car, rien n'étant supérieur au Bien, aucune cause ne précède le Bien. Le Bien serait-il alors la cause première? Non, parce que le Bien n' agit pas par lui-même; il ne peut pas exercer une action ni produire quelque chose, et l'appeler «cause» du point de vue d'un acte serait tout aussi absurde, ou du moins tout aussi inapproprié (atopon) que de considérer qu'il y ait une cause supérieure au Bien et dont l' effet transcenderait lui aussi le Bien. Par conséquent, tout étant postérieur au Bien, toutes les choses se retournant vers le Bien lui-même $(\tau \dot{\alpha}$

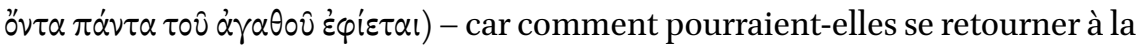
fois vers le Bien et vers une cause qui, étant supérieure au Bien, rendraient les choses elles-mêmes meilleures que le Bien ( $\tau \circ \hat{\alpha} \gamma \gamma \alpha \theta 0 \hat{\nu} \mu \alpha \dot{\lambda} \mathrm{I} \sigma \tau \alpha)$ ? -, et toutes les choses ne pouvant pas ne pas se retourner vers la cause de la totalité dont

38 Proclus, The Elements of Theology, Prop. 11: «And if the accumulation of causes may be continued to infinity, cause behind cause for ever, thus again all things will be unknowable

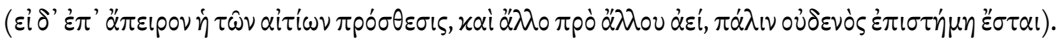
For nothing infinite can be apprehended; and the causes being unknown, there can be no

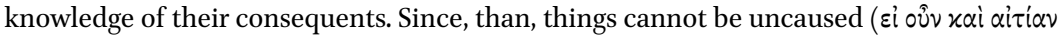

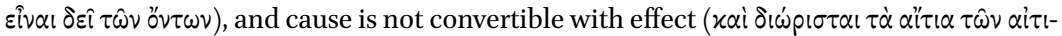

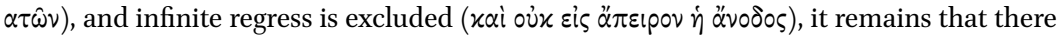

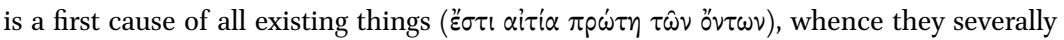

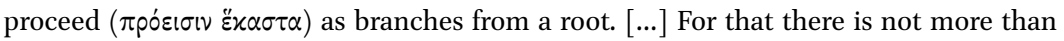
one such first principle has already been established, inasmuch as the subsistence of any

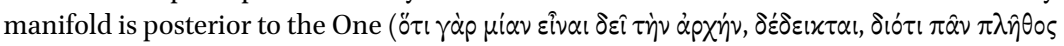

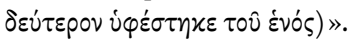

39 Proclus, The Elements of Theology, Prop. 12: «All that exists has the Good as its principium and first cause». 


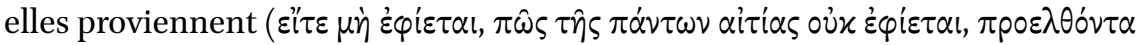
$\left.\dot{\alpha} \pi^{\prime} \alpha \dot{v} \tau \hat{\eta} \varsigma\right)$, il est alors nécessaire que le Bien dont toutes les choses dépendent

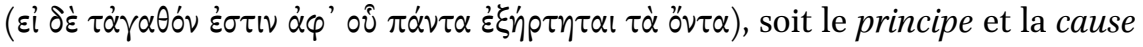

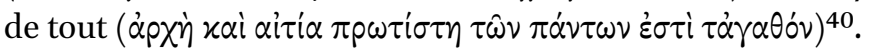

Le raisonnement de Proclus repose donc sur trois arguments qui se chevauchent dans leur circularité, et dont il faut retenir comme complémentaires d' une part l'évocation du retournement, de l'autre, la reconnaissance du Bien comme «cause » non en lui-même mais à partir du mouvement des choses vers le Bien. L'acte propre de la cause première est donc un mouvement inverse à celui de la procession: l'identité causale du Bien n'est attestée que par les choses qui retournent vers le Bien puisqu'elles en proviennent et désirent y retourner, tandis que l'identité du Bien en tant que Bien est une identité de principe. Le Bien n' a pas une identité double, mais se révèle différemment selon qu' il est pris en lui-même ou à partir de ceux qui tentent de le rejoindre en se retournant vers lui. Le retournement est évidemment le nerf de la relation; en même temps, il montre le mode de manifestation de ce rapport causal qui ne se dévoile que par le retour du causé sur/vers la cause. La supériorité est préservée de toute production qui contesterait la suprématie du Bien, de même que la supériorité de l' unité est démontrée par le retour du multiple vers une «cause première» qui ne peut qu' être unique. La procession est bien un acte, mais cet acte n'incombe qu' aux choses qui procèdent. La cause première, en tant qu' elle s'identifie au Bien, ne cause pas elle-même ce qui provient d'elle. En revanche, elle est cause du retournement des choses vers le Bien. On dirait que la «cause première» est cause sans agir, à la manière d'un aimant. Si Proclus emprunte à Aristote la critique du raisonnement par le principe qui appelle à une remonté infinie, il construit en revanche une argumentation entièrement opposée à la causalité aristotélicienne et à la nécessité de faire de la cause l'agent d'un mouvement ou d'un acte de production ou d'engendrement. À moins de considérer que le mouvement d'attrait qu' exerce le premier moteur de Métaphysique $\Lambda$ et par lequel celui-ci demeure immobile tout en produisant, comme par le désir du bien, le mouvement de tout ce qui est, soit encore un emprunt de Proclus à Aristote, directement ou par l'intermédiaire de Syrianus.

Proclus revient plusieurs fois sur cette opposition théorique en redéfinissant la cause par distinction du mouvement et des actes de production. Le com-

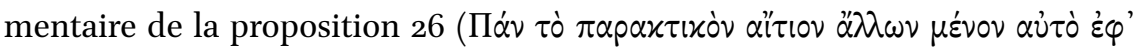

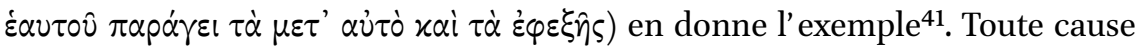

40 J'ai résumé et traduis les deux derniers paragraphes du commentaire de la proposition 12.

41 Proclus, The Elements of Theology, Prop. 26: «Every productive cause produces the next and all subsequent principles while itself remaining steadfast». 
productive produit ce qui est conséquent à cette production, mais la cause elle-même demeure impassible. La proposition 3o, déjà évoquée par Hadot, ajoute le trait caractéristique de cette causalité néoplatonicienne en précisant que même ce qui est produit de manière immédiate en procédant de la

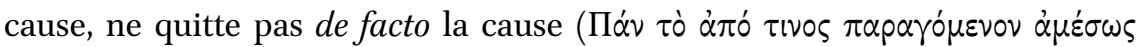

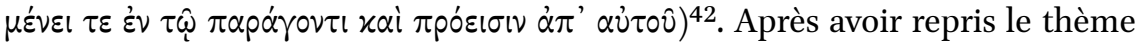
développé par les propositions 28 et 29 , à savoir que la manifestation de la procession en tant que production s'accomplit comme ressemblance $(\pi \hat{\alpha} \sigma \alpha$

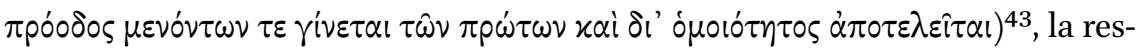
semblance transgressant la distinction et l'union et précédant nécessairement la dissemblance, le commentaire de la proposition 30 conclut qu' entre demeu-

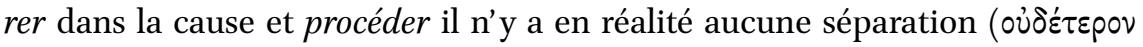

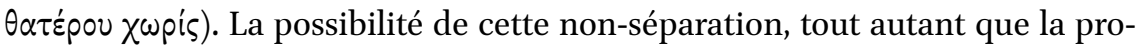
cession et le retournement reposent donc sur le rôle majeur que Proclus assigne à la homoiôsis. La ressemblance homotétique est clairement définie à la fin du commentaire de la proposition 29. On y lit que ce qui se trouve être second puisque provenant de ce qui est premier, s' accomplit comme ressemblance, car la ressemblance constitue le moyen par lequel à la fois on préserve l' identité entre l' engendré et l' engendrant, et on laisse se montrer et s' accomplir en tant

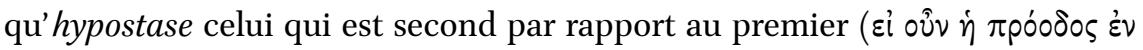

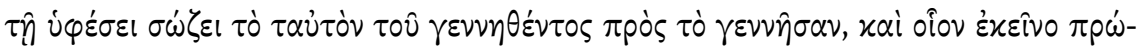

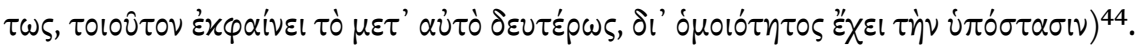
Proclus ajoute à ces affirmations que la ressemblance est inhérente à la production, puisque ce qui se produit en procédant de la cause, se produit par

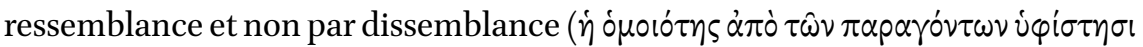

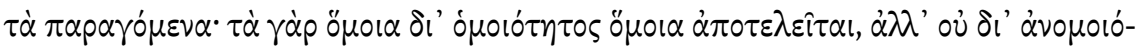
$\tau \eta \tau \circ \varsigma)^{45}$.

Dans la proposition 35 Proclus reprend une dernière fois de manière explicite la caractéristique majeure de la causalité émanant de la «cause première ». Il y affirme que l'effet reste dans la cause, procède de la cause et retourne à la cause, les trois actes étant nécessaires pour signifier simultanément l'identité

42 Proclus, The Elements of Theology, Prop. 30: «All that is immediately produced by any principle both remains in the producing cause and proceeds from it».

43 Première phrase du commentaire de la proposition 30.

44 Commentaire de la proposition 29, 8-11: «The procession, accordingly, since in declension it preserves an identity betwixt engender and engendered, and manifests by derivation in the consequent that character which the other has primitively, owes to likeness its substantive existence».

45 Commentaire de la proposition $29,6-8:$ «[... it is likeness which generates the product out of the producer: for like things are made by likeness, and not by unlikeness ». 
et la distinction de la «cause première» et du causé46. Le commentaire associe

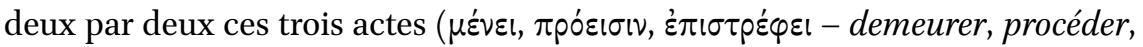
retourner) et développe les conséquences possibles et les impossibilités de la réduction de ces trois actes simultanés à deux actes contraires. Parmi les cas décrits, deux nous intéressent plus particulièrement puisque Proclus s'en sert pour stipuler la nécessité de la distinction et définir la nature de l'identité. Si, par un raisonnement absurde, le causé restait auprès de la cause en effectuant

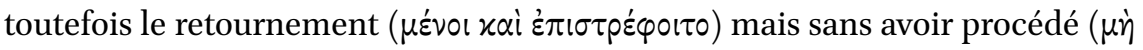
$\pi \rho \varepsilon_{p X o}(\tau 0)$, il n'y aurait alors pas de différence; or la différence est le moteur

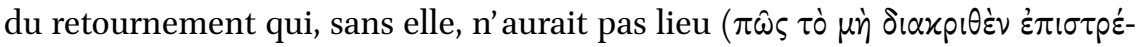

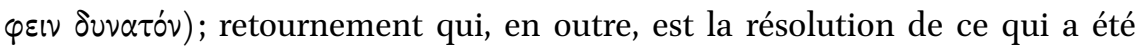

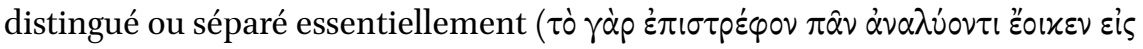

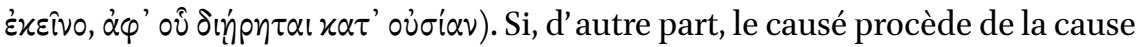

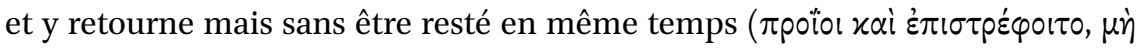

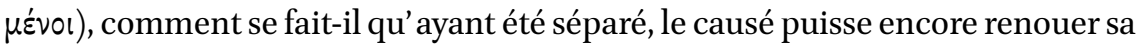

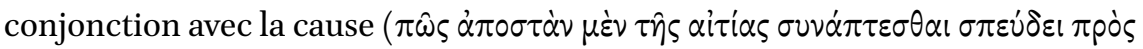
$\left.\alpha \jmath ่ \tau \nu^{\prime}\right)$, sans admettre que cette conjonction n'est possible que si elle n' ait été jamais rompue ( $\dot{\alpha} \sigma \dot{v} \nu \alpha \pi \tau o v \delta \dot{\varepsilon} \hat{\eta} \nu \pi \rho \dot{~} \tau \hat{\eta} \varsigma \dot{\alpha} \pi 0 \sigma \tau \dot{\alpha} \sigma \varepsilon \omega \varsigma$ ) et que le causé, en procédant et en y retournant, reste en même temps auprès de la cause, comme

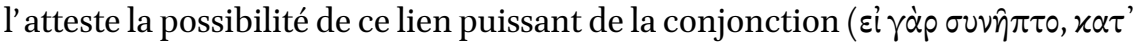

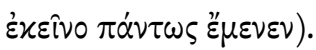

En glosant la démarche proclienne, j' ai choisi de renverser ici l'ordre des deux dernières étapes du commentaire de la proposition 35 afin de mettre en évidence le but de la démonstration ${ }^{47}$. Si la simultanéité des trois actes (demeurer, procéder, retourner) est nécessaire, et que l'on ne puisse pas réduire les trois à deux actes, réduction qui reviendrait à opposer repos et mouvement ou simplement les sens contraires d'un seul mouvement (aller/retour), la raison donnée par Proclus ne tient pas à la nature même des actes, qui sont des

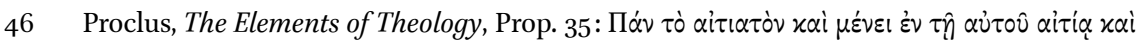

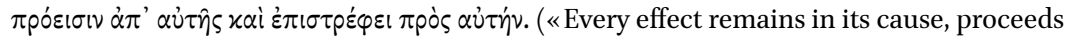
from it, and reverts upon it»).

47 Il s' agit du commentaire de la proposition 35 (18-24) que j'ai partiellement traduit en le glosant. Voici le passage dans la traduction de Dodds: «And if it should proceed and revert, but not remain, how comes it that being parted from its cause it endeavours to be conjoined with it, although before the severance there was no conjunction (since if it was conjoined with the cause it certainly remained in it)? Finally, if it should remain and revert, but not proceed, how can there be reversion without distinction (since all reversion seems to be the resolution of a principle into something from which its being divides it)?» 


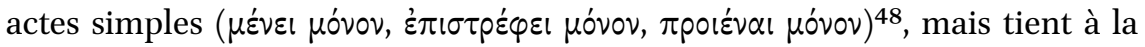
nature de l'identité. L'identité avec la «cause première» est d' ordre essentiel (ousiologique), le causé retrouvant cette identité par une conjonction (sunaptein) censée transcender la séparation ou signifier la non-séparation. Or cette conjonction, du même ordre que la sunapheia et la koinônia évoquées plus haut (proposition 32), a une importance capitale pour une double raison: d'une part elle détermine la «cause première» dont la totalité des étants procède, d' autre part elle ouvre à l'étant un accès direct à la «cause première». Ce qui procède a ainsi connaissance de ce dont il procède: une connaissance directe, par une identité de conjonction et de communion, ainsi qu' une connaissance par distinction, à travers ce qui incombe à l' étant, à savoir par les actes. Plus loin, dans les propositions 64-65 et dans leurs commentaires, Proclus étaye cette détermination de la connaissance à l' égard de l'identité essentielle de la «cause première». Parlant des degrés de causalité, il affirme que chaque monade originale donne lieu a deux séries causales: une de subsistances hypostatiques

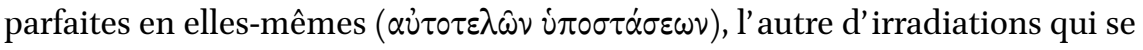

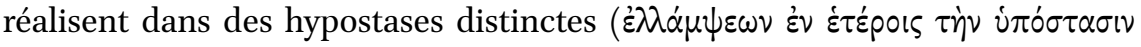

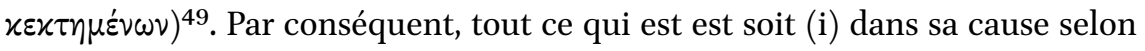
son principe formel ( $\chi \alpha \tau^{\prime} \alpha i \tau^{\prime} \alpha \nu$ है $\sigma \tau i \nu \alpha \dot{p} \chi 0 \varepsilon ı \delta \hat{\omega} \varsigma$ ), soit (ii) dans une subsistence

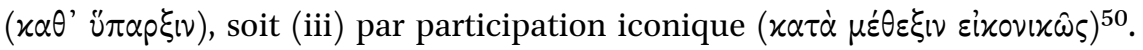
Et encore plus loin, dans la proposition 112, Proclus affirme que les premiers membres de chaque ordre ont la forme de ceux qui les précèdent ( $\tau \dot{\alpha} \pi \rho \omega \dot{\tau} \tau-$ $\sigma \tau \alpha \mu \circ \rho \varphi \eta ̀ v)$. Mais ce lien, signifié par la forme, est évidemment propre à un contexte théorique différent, en l'occurrence à ce qui procède selon l'ordre des unités (hénades), non à ce qui procède, comme la totalité, de la «cause première» unique.

\section{Remarques conclusives}

Concluons brièvement sur les possibilités d'appréhender la cause première et sur les traits spécifiques de la causalité dite «néoplatonicienne» telle qu'elle apparaît chez ces quelques auteurs évoqués. La cause première n' est cause que par ce que peut témoigner d'elle quelque chose qui en provient, mais elle-même ne donne lieu ni à une procession ni à un acte de production ou

48 Conclusion du commentaire de la proposition 35 (25).

49 Cf. Proclus, The Elements of Theology, Prop. 64.

$5^{\circ}$ Cf. Proclus, The Elements of Theology, Prop. 65. 
d' engendrement. La cause première n' est responsable que du retour vers elle de ce qui en provient, et encore, elle n' est responsable de ce retour que par délégation, puisque l'acte du retour revient à ce/celui qui retourne. On dirait que la cause première est cause comme malgré elle et qu' elle est déterminée comme cause par ce qu' elle-même n' est pas, bien qu' elle en soit l'origine. Sont ici en cause (si on peut dire) tout autant la notion de «cause» que sa désignation de «première». Le fait d'être cause de ce qu' elle n'est pas satisfait au principe de séparation de ce qui demeure impassible et justifie sa proximité immédiate avec l'Un ou le Bien. Saloustios et Proclus abondent dans ce sens. Pour Victorinus en revanche, qui doit prouver la consubstantialité du Père et du Fils-Logos, la causalité en général et le statut particulier d'une «cause première » soulèvent des difficultés; le théologien se voit obligé de recourir à une relation de causalité réciproque entre les hypostases et de marquer la différence par l'introduction d'une «précause».

Est en outre récurrente pour signifier la cause première sa qualification d'acte qui n'agit pas, aussi bien chez Proclus, que chez Plotin, Victorinus ou Saloustios. Porphyre propose l'image de la source qui fait refluer vers ellemême ce qu' elle possède (Sentence 44). En raison de ce paradoxe d'acte qui n'agit pas, la cause première est associée au principe mais sans être identifiée à un principe, comme elle n' est pas identifiée non plus au Bien lui-même. Saloustios lui attribue une nature bonne et justifie ainsi le retour vers elle comme le mouvement d'un désir du meilleur. La nécessité de saisir le moyen ou le mode par lequel une cause agit sans agir, puisqu' elle est supérieure à l'acte, a donné lieu à deux interprétations: l' une par la procession, assortie du retournement et de la manence (Porphyre, Proclus), l'autre par un usage du couple acte-puissance, avec un renversement de la prééminence de l'acte au profit de la puissance (Victorinus, Saloustios). Mais dans les deux interprétations une justification topique semble s'imposer: l' acte est dans la puissance; ce qui procède peut y retourner car il était resté tout en procédant; le multiple est contenu par l' unité; l' intelligé est dans l'intellect, etc. Sur cette même justification topique repose aussi la compréhension de la totalité. La cause première est cause du tout; on devrait même dire qu' elle n' est cause que du tout, tout s' entendant à la fois comme totalité et comme unité.

Enfin, la cause première n' apparaît ni comme une cause essentielle (en tant que première elle est qualifiée de huperousia par Saloustios), ni comme une cause formelle (elle pourrait tout au plus être considérée comme principe formel, arkhoeidôs de ce qui est causé, dit Proclus, mais elle n'est pas forme en elle-même). Cependant, la cause première peut être connue. Elle est connue par ce qui demeure, par la non-séparation d' elle-même et par la communion qui s' engage entre la cause et ce qui retourne à la cause ayant effectué la pro- 
cession et boucler le retour. En somme, la cause première est connue sous deux modalités: par les actes qui incombent à ce qui procède et y retourne, et par la conjonction-communion (sunaphê, sunaptein, koinônia) qui a lieu dans l'immanence de la cause elle-même. On retrouve dans le modèle de cette connaissance que décrivent aussi bien Saloustios que Proclus, la connaissance trinitaire que décrivent Victorinus et en partie Basile de Césarée quand ils parlent de la distinction individualisante des opérations propres aux hypostases de la Trinité et de la connaissance réciproque que les hypostases ont entre elles en se reflétant les unes dans les autres de manière iconique et en réalisant ainsi la conjonction et la communion intime parfaite de la Trinité. Bien que Proclus procède par déduction pour démontrer l' existence de la cause première et établir son statut à l'égard du causé et sa séparation à l'égard de tout acte de production, engendrement ou participation, la connaissance que la cause donne d'elle-même au causé n' est pas de l'ordre d'une déduction ou d'une induction formelle, mais se réalise comme saisie ou appréhension et s' avère immédiate. La cause première étant en dehors de la temporalité, sa connaissance est nécessairement non discursive, ne donnant lieu ni à un jugement par antérieur et postérieur, ni à une distinction d'ordre ontologique (substance, espèce, individu). Cette connaissance, que l'on qualifierait volontiers de saisie par vision, répond à la capacité de la cause première de se laisser connaître uniquement dans l'image que l'acte, auquel elle ne s'identifie pas, donne d' elle tout en préservant son caractère strictement aporétique.

\section{Bibliographie}

\section{Sources primaires}

Aristote, Métaphysique, Loeb Classical Library, Harvard University Press, 1933; trad. fr. J. Tricot, Paris, Vrin, 1991.

Marius Victorinus, Traités théologiques sur la Trinité, éd. P. Henry, trad. P. Hadot, Paris, Cerf, 196o.

Numénius, Fragments, trad. É. des Places, Paris, Les Belles Lettres, 1973.

Philon d' Alexandrie, De ceternitate mundi, trad. J. Pouilloux, Paris, Cerf, 1969.

Platon, Timée, trad. A. Rivaud, Paris, Les Belles Lettres, 2002.

Plotin, Ennéade IV, 8 [6], 6, trad. L. Lavaud, Paris, GF, 2002 ; Ennéade v, 6 [24], 6, trad. L. Lavaud, Paris, GF, 2004; Ennéade V, 3, 49, 12, trad. F. Fronterotta, Paris, GF, 2009. Porphyre, Sentences, Paris, Vrin, 2005.

Proclus, The Elements of Theology, éd., trad., introd. et commentaire par E.R. Dodds, Oxford, Clarendon Press, 1963. 
Proclus, Théologie platonicienne, éd. et trad. H.D. Saffrey et L.G. Westerink, Paris, Les

Belles Lettres, 2003.

Saloustios, Des dieux et du monde, trad. G. Rochefort, Paris, Les Belles Lettres, 196o.

\section{Sources secondaires}

Abramowski, L. (2005), «Nicänismus und Gnosis im Rom des Bischofs Liberius: Der Fall des Marius Victorinus », dans Zeitschrift für antikes Christentum 8, p. 513-566.

Bussanich, J. (1996), «Plotinus's Metaphysics of the One», dans L.P. Gerson (éd.), The Cambridge Companion to Plotinus, CUP, p. 38-65.

Chiaradonna, R. (2014), «Causalité et hiérarchie métaphysique dans le néoplatonisme: Plotin, Porphyre, Jamblique», dans Chôra 12, p. 67-85.

D’Ancona, C. (1996), «Plotinus and Later Platonic Philosophers on the Causality of the First Principle», dans L.P. Gerson (éd.), The Cambridge Companion to Plotinus, CuP, p. 38-65, p. 356-385

Emilsson, E.K. (1999), «The Relation between the One and Intellect in Plotinus », dans J.J. Cleary (éd.), Tradition of Platonism. Essays in Honour of John Dillon, London, Ashgate.

Emilsson, E.K. (2007), Plotinus on Intellect, Oxford, Clarendon Press.

Ferrari, F. (2007), «Separazione asimmetrica e causalità eidetica nel Timeo », dans L.M. Napolitano-Valditara (éd.), La sapienza di Timeo. Riflessioni in margine al Timeo di Platone, Milano, Vita et Pensiero, p. 147-172.

Ferrari, F. (2010), «Dinamismo causale e separazione asimmetrica in Platone», dans F. Fronterotta (éd.), La scienza e le cause a partire dalla Metafisica di Aristotele, Naples, Bibliopolis, p. 33-72.

Ferrari, F. (2015), «Asimmetria e non-reversibilità nella metafisica di Platone», dans R. Radice, G. Tiengo (eds), Seconda navigazione. Omaggio a Giovanni Reale, Milano, Vita e Pensiero, p. 219-232.

Hadot (1968), Porphyre et Victorinus, Paris, Études augustiniennes, 1968.

Michalewski, A. (2014), La puissance de l'intelligible. La théorie plotinienne des Formes au miroir de l'héritage médioplatonicien, Leuven, Leuven University Press.

Rutten, C. (1956), «La doctrine des deux actes dans la philosophie de Plotin», dans Revue philosophique, 146, p. 100-106.

Tardieu, M. (1996), «Recherches sur la formation de l'Apocalypse de Zostrien et les sources de Marius Victorinus », dans Res Orientales 9, Bures-sur-Yvette.

Trambrun-Krasker, B. (2006), Plethon, le retour de Platon, Paris, Vrin.

Vasiliu, A. (2014a), «Relation absolue et relation relative. La Trinité selon Marius Victorinus et Basile de Césarée», dans Quaestio 13, p. 73-101.

Vasiliu, A. (2014b), «Note liminaire. Des principes et des causes», dans Chôra 12, p. 5-9. 